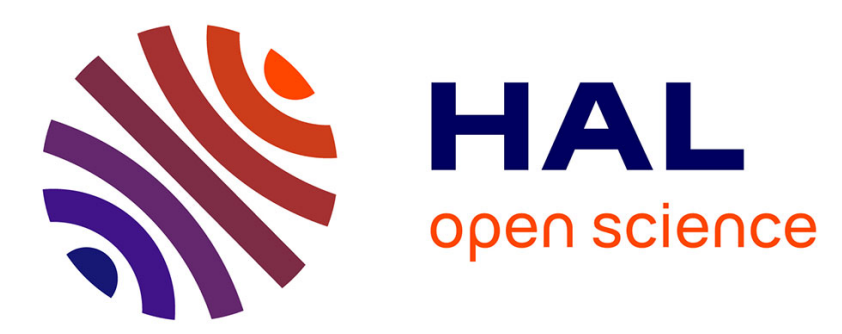

\title{
Characterization of the ER-located zinc transporter ZnT1 and identification of a vesicular zinc storage compartment in Hebeloma cylindrosporum
}

Damien Blaudez, Michel Chalot

\section{- To cite this version:}

Damien Blaudez, Michel Chalot. Characterization of the ER-located zinc transporter ZnT1 and identification of a vesicular zinc storage compartment in Hebeloma cylindrosporum. Fungal Genetics and Biology, 2011, 48 (5), pp.496-503. 10.1016/j.fgb.2010.11.007 . hal-02372451

\section{HAL Id: hal-02372451 \\ https://hal.science/hal-02372451}

Submitted on 8 Apr 2020

HAL is a multi-disciplinary open access archive for the deposit and dissemination of scientific research documents, whether they are published or not. The documents may come from teaching and research institutions in France or abroad, or from public or private research centers.
L'archive ouverte pluridisciplinaire HAL, est destinée au dépôt et à la diffusion de documents scientifiques de niveau recherche, publiés ou non, émanant des établissements d'enseignement et de recherche français ou étrangers, des laboratoires publics ou privés. 


\title{
Characterization of the ER-located zinc transporter ZnT1 and identification of a vesicular zinc storage compartment in Hebeloma cylindrosporum
}

\author{
Damien Blaudez, Michel Chalot
}

UMR INRA/UHP 1136 “Tree-microbe Interactions”, Faculty of Sciences and Technology, Nancy-University, BP 70239, F-54506 Vandoeuvre-les-Nancy, France

\begin{abstract}
Metal tolerance of filamentous fungi is a poorly understood mechanism. In order to unravel the molecular basis of zinc (Zn) tolerance in the ectomycorrhizal fungal model Hebeloma cylindrosporum, we carried out a functional screening of an H. cylindrosporum cDNA library in the zrc1D mutant strain of Saccharomyces cerevisiae to search for genes conferring Zn tolerance to yeast cells. This strategy allowed the isolation of HcZnT1, a gene belonging to the cation diffusion facilitator family, which induced tolerance to Zn, but not to other metals. HcZnT1 was constitutively expressed in Hebeloma cells, whatever the Zn status of the medium and the fungal cell type (mycelia, sporocarps, mycorrhizas). A HcZnT1:GFP fusion protein was expressed in yeast and the corresponding fluorescence was recorded on endoplasmic reticulum mem- branes. Taken together, these different findings suggest a dual role of HcZnT1 in Zn homeostasis of fungal cells, by supplying requested Zn ions for the functioning of the endoplasmic reticulum as well as by detoxifying the cytosol under Zn stress. Zn pools were also investigated by using the Zn-specific fluoro- phore zinquin in H. cylindrosporum cells. Zinquin labeling revealed compartmentalization in intracellular vesicles interspersed throughout the cytoplasm that do not correspond to vacuolar compartments. Alto- gether the present data represent the first steps into the understanding of Zn homeostasis and tolerance in Hebeloma.
\end{abstract}

\section{Introduction}

Several studies have focused on benefits of symbioses for the plant hosts growing on trace elements (TE) contaminated sites, with the aim of possible biotechnological applications such as phytoremediation (Gadd, 2010; Kozdroj et al., 2007; Krupa and Kozdroj, 2004, 2007). It is now widely accepted that rhizospheric microorganisms may actively participate to phytoremediation processes, and may be useful in extending the application of phytoremediation to additional trace element contaminated sites (Kuffner et al., 2008). Hosting different mycorrhizal types might be of functional importance for plant nutrition (Smith and Read, 2008) and has been shown to contribute to metal tolerance of host plants as fungi can reduce the metal uptake by the plant by seques- tration, extracellular precipitation and biosorption to the cell walls (Bellion et al., 2006; Blaudez et al., 2000a; Hildebrandt et al., 2007). Mechanisms have been detailed in different mycorrhizal fungi, including induction of metallothionein (Bellion et al., 2007; Ramesh et al., 2009), superoxide dismutase (Jacob et al., 2001; Vallino et al., 2009) or thiol compounds (Courbot et al., 2004). Using radiotracer flux analyses, the significant accumulation of Cd found in the vacuolar compartment has been suggested as an essential Cd detoxification mechanism in the ectomycorrhizal fun- gus Paxillus involutus (Blaudez et al., 2000b). Indeed, a number of recent data support the hypothesis that mycorrhizal fungi play a filtering/sequestering role on plant roots (Arriagada et al., 2010; Bissonnette et al., 2009; Carvalho et al., 2006; Christie et al., 2004; Cobbett and Goldsbrough, 2002; Colpaert et al., 2004; Lee et al., 2003; Mathur et al., 2007), enhancing root to shoot metal ratio and increasing survival rate in harsh conditions (Leung et al., 2007).

However, there are only few reports on transport mechanisms in mycorrhizal fungi, and even less information on TE transporters. Specific transporters, encoded by multigenic families, are responsi- ble for the uptake and secretion of metal ions, and for their sequestration into organelles (Gaither and Eide, 2001; Hall and Williams, 2003; Kambe et al., 2004; Nies, 2003). Cation diffusion facilitator (CDF, TC 2.A.4) transporters, first identified in bacteria (Nies and Silver, 1995), are ubiquitous, spanning all three kingdoms of life: Archaea, Eubacteria and Eukaryotes (Montanini et al., 2007). The majority of CDF proteins possess six putative transmembrane domains (TMDs), with the $\mathrm{N}$ and $\mathrm{C}$ termini cytoplasmic, as experi- mentally demonstrated for bacterial members (Anton et al., 1999; Wei and $\mathrm{Fu}, 2005$ ). A signature sequence between TMDs I and II was proposed in 1997 (Paulsen and Saier, 1997) and modi- fied in 2007 (Montanini et al., 2007). A characteristic C-terminal cation efflux domain is present in all members (Maser et al., 2001). Most CDF transporters also contain a histidine-rich region, either between TMDs IV and V, or at the $\mathrm{N}$ and $\mathrm{C}$ termini. Such regions are predicted to be cytoplasmic, cis to metal uptake, and could function as potential metal $\left(\mathrm{Zn}^{2+}, \mathrm{Co}^{2+}\right.$, and/or $\left.\mathrm{Cd}^{2+}\right)$ binding do- mains. However the ER-localized Zrg17, a $\mathrm{Zn}^{2+}$ transporter recently characterized in yeast, displays a histidine-rich loop predicted to reside between TMDs III and IV, toward the ER lumen (Ellis et al., 2005). CDF transporters are postulated to be involved exclusively in transport of metals into intracellular vesicles or out of the cell across the plasma membrane (Palmiter and Huang, 2004). The Sac- charomyces cerevisiae Zrc1 (MacDiarmid et al., 2000) and Cot1 (Conklin et al., 1992) are located on vacuolar membranes, whereas the Schizosaccharomyces pombe Zhf1 (Clemens et al., 2002) and S. cerevisiae Zrg17 (Ellis et al., 2005) are located on ER membranes.

CDF-like sequences in mycorrhizal fungi have been obtained in the course of expressed sequence tag projects (Stommel et al., 2001) and a gene coding for a putative CDF has been isolated and characterized from the arbuscular mycorrhizal (AM) fungus Glomus intraradices (Gonzalez-Guerrero et al., 2005). Here we report the isolation and functional characterization of the HcZnT1 gene coding for a CDF Zn transporter in Hebeloma cylindrosporum, as well as the visualization of zinc pools by fluorescent tracer.

\section{Materials and methods}

\subsection{Yeast strains, fungal materials and media}

The S. cerevisiae strains used for heterologous expression of HcZnT1 were BY4741 (MATa his3D1 leu2D0 met15D0 ura3D0), zrc1D (MATa his3D1 leu2D0 met15D0 ura3D0 ZRC1::kanMX4), cot1D (MATa his3D1 leu2D0 met15D0 ura3D0 CoT1::kanMX4), ycf1D (MATa his3D1 leu2D0 met15D0 ura3D0 YCF1::kanMX4) and pmr1D (MATa his3D1 leu2D0 met15D0 ura3D0 PMR1::kan- MX4). The four mutants used derived from the parent strain BY4741 and they were all obtained from Euroscarf (http://www. uni- 
frankfurt.de/fb15/mikro/euroscarf/). Synthetic yeast medium without uracil (yeast nitrogen base (Sigma Aldrich, Lyon, France) 6,7 $\mathrm{g}^{1}, 2 \%$ glucose, drop out (Sigma) $1.92 \mathrm{~g} \mathrm{I}^{1}$ ) was used. For me- tal tolerance assays, yeast was grown in liquid SD until $0 D_{600 \mathrm{~nm}}=1$. These cultures were subsequently used to inoculate SD control plates (no extra metal added) or SD plates containing toxic concentrations of zinc, cadmium, manganese, or cobalt. Yeast cells expressing HcZnT1::GFP under the control of the Gal1-induc- ible promoter were grown in liquid SD medium containing $2 \%(\mathrm{w} / \mathrm{v})$ galactose instead of glucose.

The ectomycorrhizal fungus H. cylindrosporum Romagnesi (hap- loid strain h1) was grown on cellophane-covered agar medium as previously described (Javelle et al., 2003). H. cylindrosporum-Pinus pinaster mycorrhizas and sporocarps were collected from plants, inoculated with the dikaryotic strain D2, growing in a greenhouse. Material was frozen in liquid nitrogen and stored at $80^{\circ} \mathrm{C}$ until use.

\subsection{Library screening}

To identify ectomycorrhizal fungal cDNAs able to increase zinc tolerance, the zrc1D yeast strain was transformed with a H. cylindrosporum cDNA library kindly provided by H. Sentenac (UMR 5004 Agro-M-CNRS-INRA-UM2, Montpellier, France). The library was constructed in the pFL61 yeast expression vector under the control of the strong constitutive PGK promoter (Lambilliotte et al., 2004). Transformations were performed by the standard lith- ium acetate method (Gietz et al., 1992). $\mathrm{Zn}^{2+}$-tolerant Ura ${ }^{+}$trans- formants were selected after plating the transformants on SD

medium supplemented with the appropriate amino acids and con- taining $20 \mathrm{mM} \mathrm{ZnCl}_{2}$. Recombinant pFL61 plasmids that conferred zinc tolerance were isolated by the Zymoprep Yeast Plasmid Mini- prep kit (Proteigene, Saint Marcel, France) by following the manufacturer's recommendations. Plasmids were subsequently used in retransformation experiments of zrc1D to confirm the functional tolerance before sequencing. The amino acid sequence of HcZnT1 was deduced from the corresponding cDNA sequence and was used in BLAST searches to find the closest fungal orthologs in databases. Amino acid sequences of previously studied fungal orthologs of the same family were also retrieved from databases and were aligned and edited by the multiple sequence alignment editor and shading utility GeneDoc software (version 2.6.003) (http://www.psc.edu/ biomed/genedoc).

\subsection{Construction of GFP fusions for expression in yeast}

To construct HcZnT1::GFP, the full-coding sequence of HcZnT1 was amplified from the plasmid pFL61-HcZnT1 with the primers HcZnT1f $\left(5^{0}\right.$-CCCCGGATCCATGAAGAATACAACCAAGATA- $\left.3^{0}\right)$ and HcZnT1r $\left(5^{0}\right.$-CCCCGAATTCAGCTGCATCGCCGACCTTTAT- $\left.3^{0}\right)$, introducing a BamHI site and a EcoRI site at the $5^{0}$ and $3^{0}$ end, respectively (sites underlined), and cloned into the BamHI-EcoRI-digested pYES2-GFP plasmid (Blaudez et al., 2003). A similar procedure was used to produce the HcAMT1::GFP fusion. HcAMT1 was sub-cloned into the HindIII-BamHI-digested pYES2-GFP plasmid after PCR amplification and HindIII/BamHI digestion. The primers used for HcAMT1 amplification were HcAMT1f $\left(5^{0}\right.$-CCCAAGCTTATGGTCAACG TCACATACGAT- $\left.{ }^{0}\right)$ and HcAMT1r $\quad\left(5^{0}\right.$ CCCGGATCCCCTGCTGATTTCTC TTCTACGCT- $3^{0}$ ).

\subsection{Fluorescence microscopy}

Yeast vacuolar membranes were stained with FM4-64 (Molecu- lar Probes, Heyden, The Netherlands) as previously described (Blaudez et al., 2003). Small aliquot (5 ll) of the resulting cell sus- pension was mounted on glass slides and viewed with a fluorescence microscope. A Nikon Optiphot-2 fluorescence microscope equipped with a Nikon D1 camera was used to document results. For both FM4-64 and GFP-deriving fluorescence, a filter was used to select the wavelength of the excitation light to the $475-500 \mathrm{~nm}$ band, and the fluorescence emission was recorded above $520 \mathrm{~nm}$. The staining of yeast nuclei was performed by incubating fungal cells with $1 \mathrm{lg} / \mathrm{ml}$ DAPI for $10 \mathrm{~min}$ and in this case a UV fil- ter was used. For Zn staining, H. cylindrosporum was grown for 1 week on solid NP medium (Javelle et al., 2003) enriched with $200 \mathrm{lM} \mathrm{ZnCl}_{2}$. Preliminary experiments revealed that this concen- tration was a sub-toxic condition for H. cylindrosporum growth. Mycelia were collected and incubated for $30 \mathrm{~min}$ in NP medium containing 18 lM zinquin (Biotium, Inc., Hayward, CA), a zinc-spe- cific fluorescent marker (Mahadevan et al., 1996). Cells were briefly rinsed in NP medium to remove medium-derived fluorescence and cells were finally analysed by fluorescence microscopy by using a UV filter (excitation band: $300-400 \mathrm{~nm}$, emission band $>420 \mathrm{~nm}$ ). The fluorescence pattern was identical in most cells, indicating a uniform staining of the mycelia by the probe. For staining the lu- men of the fungal vacuoles, mycelia were incubated in NP medium for 20 min with $10 \mathrm{~lm}$ carboxy-DCFDA (Molecular Probes, Heyden, The Netherlands), rinsed and viewed with a fluorescent microscope and with the same filter as used for GFP observation.

\subsection{HcZnT1 expression}

Total RNAs from free-living fungus, mycorrhizas and sporocarps were extracted by using the Qiagen RNA plant extraction kit with addition of RNase-free DNase (Promega, Madison, WI) to avoid genomic DNA contamination. To study the effect of external $\mathrm{Zn}$ concentrations on HcZnT1 expression, mycelia were also exposed for $18 \mathrm{~h}$ to a range of $\mathrm{Zn}\left(0.3 ; 3 ; 30 ; 300\right.$ and $\left.3000 \mathrm{~lm} \mathrm{ZnSO}_{4}\right)$. Reverse transcription (RT) reactions were performed with $0.5 \mathrm{lg}$ total RNA and with the enzyme Omniscript (Qiagen, Hilden, Germany) as recommended by the manufacturer. RT reactions were performed for $1 \mathrm{~h}$ at $37{ }^{\circ} \mathrm{C}$. Each PCR reaction contained $0.15 \mathrm{U} / \mathrm{ll}$ Taq polymer-ase (Biolabs), 1 buffer, $0.2 \mathrm{mM} \mathrm{dNTP,} 25 \mathrm{ng}$ of RT products, and $200 \mathrm{nM}$ of adequate primers. The PCR primers used were the follow- ing: HcZnT1RTf $\left(5^{0}\right.$-ATTGGCTTGGGATTGAATATCGTCA- $\left.3^{0}\right)$ and HcZnT1RTr $\left(5^{0}\right.$-TCACTTTCGCGAGGTCGAGATGTAT- $3^{0}$ ) for HcZnT1 amplification, HcGSf $\left(5^{0}\right.$-GGCACCCCGAACAGGACCAA- $\left.3^{0}\right)$ and HcGSr $\left(5^{0}\right.$-GTAGTAGGGTCCTTGGGGAC- $\left.3^{0}\right)$ for HcGS amplification, and HcUbqf $\left(5^{0}\right.$-AGATCCAAGACAAAGAGGGCAT- $\left.3^{\circ}\right)$ and HcUbqr $\left(5^{0}\right.$-GG GATTCCTTCCTTATCTTGAA- $\left.3^{\circ}\right)$ for HcUbq amplification. HcGS (accession number: AF411820) and HcUbq (accession number: BU964130) encode the glutamine synthetase and 
a ubiquitin from $\mathrm{H}$. cylindrosporum, respectively and were used as reference genes. The products were amplified by PCR with a Mastercycle (Eppen- dorf, Le Pecq, France) under the following conditions: $94^{\circ} \mathrm{C}$ for $1 \mathrm{~min}$, followed by 30 cycles at $94^{\circ} \mathrm{C}$ for $30 \mathrm{~s}, 60^{\circ} \mathrm{C}$ for $30 \mathrm{~s}$ and $72{ }^{\circ} \mathrm{C}$ for $30 \mathrm{~s}$. PCR samples were taken at successive cycles and analysed by agarose gel electrophoresis for quantification, check- ing that amplification did not reach saturation. Final quantitative analyses were realized after 27 (HcGS and HcUbq) or 32 (HcZnT1) PCR cycles, respectively. Three independent experiments were conducted and gave similar results.

3. Results

\subsection{Isolation of H. cylindrosporum ZnT1 cDNA}

A H. cylindrosporum cDNA library was screened in yeast $\mathrm{zrc1D}$ for $\mathrm{Zn}^{2+}$ tolerance on agar medium containing $20 \mathrm{mM} \mathrm{ZnCl}_{2}$. Dele- tion of the Zrc1 gene, which encodes a transporter responsible to sequester Zn into the vacuole, increased the mutant sensitivity to- wards Zn (Kamizono et al., 1989; Li and Kaplan, 1998). Colonies were selected for their capability to grow vigorously on the $\mathrm{Zn}^{2+}$ - toxic medium. Plasmids were isolated and transformed into the zrc1D strain and re-screened on $\mathrm{Zn}^{2+}$-toxic medium in order to confirm that expression of fungal cDNAs is responsible for the $\mathrm{Zn}^{2+}$ tolerance phenotype. Among 15 clones, restriction digestion and sequencing showed that all the cDNAs were identical and en- coded a protein with features similar to the CDF (cation diffusion facilitator) transporter family. BLASTP and BLASTX searches in pub- lic databases of the H. cylindrosporum protein showed a strong sim- ilarity with putative CDF genes/proteins from the basidiomycete fungi Laccaria bicolor, Coprinopsis cinerea, Lentinula edodes, and Schizophyllum commune. However the function of these orthologs remains putative since none of the corresponding genes have yet been functionally characterized. Conversely, GintZnT1 (Accession No. CAE00445) from G. intraradices, SpZhf1 (Accession No. NP_593645) from S. pombe, ScZrc1 (Accession No. CAA88653), and ScCot1 (Accession No. CAA99636) from S. cerevisiae, encoding for already characterized Zn-CDF subfamily proteins (Montanini et al., 2007). The protein sequences of these four characterized CDF were therefore used for sequence comparison with HcZnT1 (Fig. 1A and B). The HcZnT1 cDNA encodes a protein of 369 amino acids with a predicted molecular weight of $40 \mathrm{kDa}$. Analysis of the $\mathrm{H}$. cylindrosporum sequence shows that it contains the complete $\mathrm{N}$-terminal signature sequence for the CDF family and also has a histidine-rich region (Fig. 1A) that is commonly found in the subgroup III members of the CDF family (Blaudez et al., 2003; Montanini et al., 2007). The HcZnT1 sequence also possesses a cation efflux domain indicative of the CDF family as identified by the Pfam protein domain database (http://pfam.wustl.edu). Furthermore, prediction of TMDs by the TMMOD programme (http:// liao.cis.udel.edu/website/servers/TMMOD/scripts/frame.php?p= submit) identified a model for HcZnT1 consisting of 6 membrane spanning regions (Fig. 1A). HcZnT1 also contains the typical HxxxD motif on TMD I and TMD V (Fig. 1A) that revealed its zinc specificity (Montanini et al., 2007). The H. cylindrosporum HcZnT1 sequence was deposited in GenBank database with the accession number DQ515992.

A
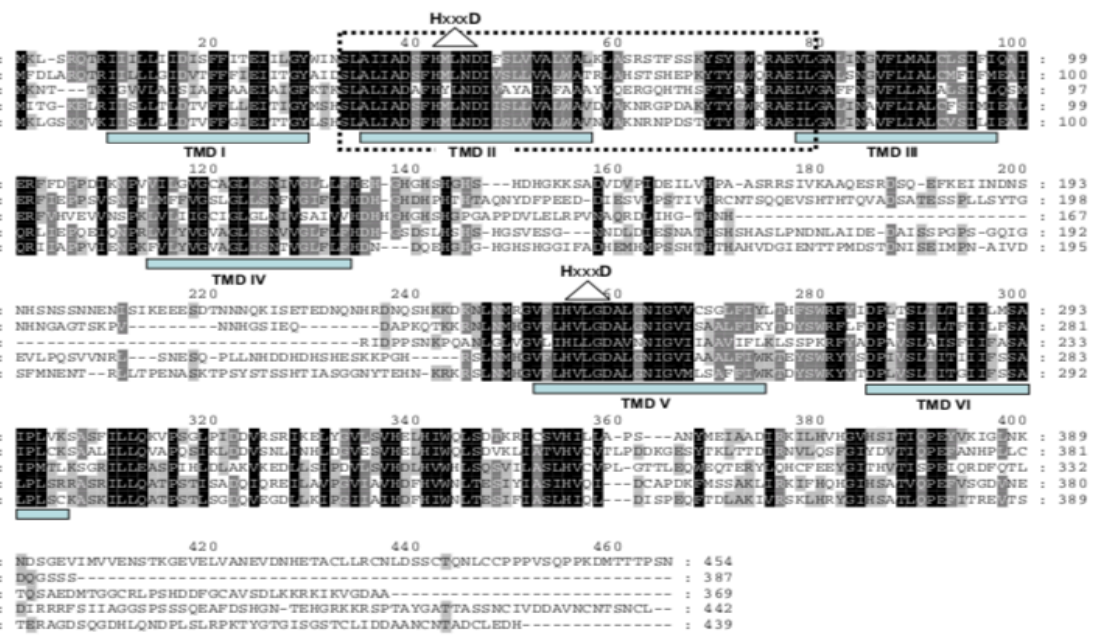

B

\begin{tabular}{lccccc}
\hline & GZnT1 & SpZHF1 & HCZnT1 & ScZRC1 & ScCOT1 \\
\hline GZnT1 & - & 40 & 26 & 37 & 37 \\
SpZHF1 & & - & 29 & 36 & 36 \\
HeZnT1 & & - & 26 & 27 \\
ScZRC1 & & & - & 52 \\
\hline
\end{tabular}

Fig. 1. Sequence comparison of functionally characterized Zn-CDF transporters from various fungal species. (A) Alignment of HcZnT1 with GintZnT1 (Accession No. CAE00445), SpZhf1 (Accession No. NP_593645), ScZrc1 (Accession No. CAA88653), and ScCot1 (Accession No. CAA99636) protein sequences. Amino acid residues that are conserved in all five sequences, or in at least two of them are shown on a black or gray background, respectively. The positions of predicted transmembrane domains (TMDs) are underlined in blue. Location of the CDF signature is shown by a dotted box. (B) Percent similarity between Zn-CDF transporters from the fungal species (TMDs) are underit
described in A.

\subsection{Metal tolerance in yeast expressing HcZnT1 cDNA}

HcZnT1 cDNA was expressed in S. cerevisiae mutant strains that are unable to grow on high concentration of various metals, and growth was monitored on either control or metal-supplemented medium. The wild-type strain was able to grow at high $\mathrm{Zn}$ concentration (Fig. 2A and B), whereas deletion of either the Cot1 or the Zrc1 gene, which encode transporters that sequesters Zn into the vacuole, rendered both mutants highly sensitive to Zn (Kamizono et al., 1989 ; Li and Kaplan, 1998). The Zn-sensitive phenotype of both Cot1D and Zrc1D mutants was fully complemented by HcZnT1 on $15 \mathrm{mM}$ Zn (Fig. 2A and B). Cot1D mutant is also sensi- tive to high Co concentrations (Conklin et al., 1992), however HcZnT1 did not complement this phenotype on 2mM Co (Fig. 2A). We further 
expressed HcZnT1 in the pmr1D and ycf1D mutant strains, which are unable to grow on Mn and Cd, respec- tively (Li and Kaplan, 1997 ; Ton et al., 2002). Transformation of the mutant strains with HcZnT1 did not restore growth of pmr1D on 3 mM Mn (Fig. 2C) nor that of ycf1D on $0.1 \mathrm{mM} \mathrm{Cd}$ (Fig. 2D). The data obtained with the different mutant strains suggest that HcZnT1 specifically transports $\mathrm{Zn}$ but not $\mathrm{Co}, \mathrm{Mn}$, nor Cd.

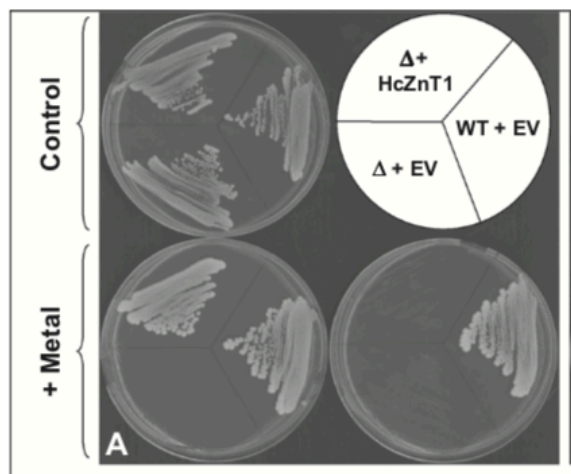

$15 \mathrm{mM} Z \mathrm{n}$

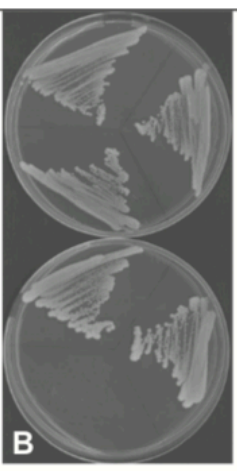

$15 \mathrm{mM} \mathrm{Zn}$

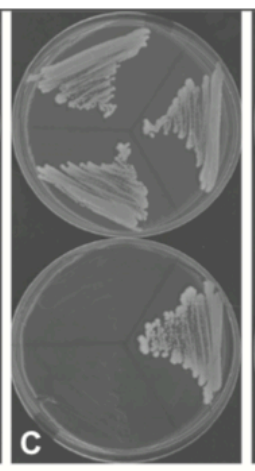

$3 \mathrm{mM} \mathrm{Mn}$

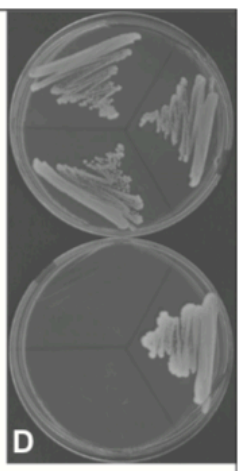

$0.1 \mathrm{mM} \mathrm{Cd}$

Fig. 2. Complementation of yeast mutants on selective media. Plates with (lower panel) or without (upper panel) added metals were streaked with the wild-type S. cerevisiae strain BY4741 transformed with pFL61 empty vector (WT + EV), with mutant strains transformed with pFL61 empty vector (D + EV) or with pFL61 containing HcZnT1 (D + HcZnT1). The mutant strains used are cot1D (A), zrc1D (B), pmr1D (C) and ycf1D (D). Plates were incubated at $30^{\circ} \mathrm{C}$ for 3 days. A representative plate (out of four) is shown and the experiment was carried out twice with similar results.

\subsection{HcZnT1 localization in yeast}

Considering that HcZnT1 suppressed the zrc1D phenotype and that it possesses numerous predicted transmembrane domains, HcZnT1 acts as a Zn efflux system from the cytosol, either by extruding Zn out of the cell or by transporting Zn into intracellular compartments. We therefore constructed a HcZnT1:GFP fusion protein to determine the intracellular localization of HcZnT1 more precisely. Because the HcZnT1:GFP fusion gene complemented the zinc sensitivity of the zrc1D mutant (data not shown), the fusion protein was confirmed to retain its original function. GFP fluores- cence was found at the periphery of the cell, as well as forming a central ring-like pattern (Fig. 3A). This pattern is typical of endo- plasmic reticulum (ER) localization in yeast. The central ring pattern indeed corresponds to ER structures surrounding the nucleus, whereas the fluorescence at the cell periphery represents strands (Fig. 3A and E, arrows) connecting the nucleus to periphe- ral ER structures. This ER localization was confirmed after nuclei staining with DAPI which indeed revealed that GFP surrounded the nuclei (Fig 3G). Undoubtedly, GFP was not present on the vac- uolar membrane as demonstrated by the lack of co-localization be- tween GFP (green) and FM4-64 (red) fluorescence patterns. The lipophilic dye FM4-64 is used in yeast to specifically stain the vac- uolar membrane (Vida and Emr, 1995). Considering the discontinuous GFP pattern at the cell periphery, the localization at the plasmalemma can also be ruled out. Instead, HcAMT1 (an ammo- nium transporter from H. cylindrosporum; Javelle et al., 2003) was used as a control to illustrate typical plasma membrane staining (Fig. 3C). From these experiments, HcZnT1 therefore appears to be localized on ER membranes.
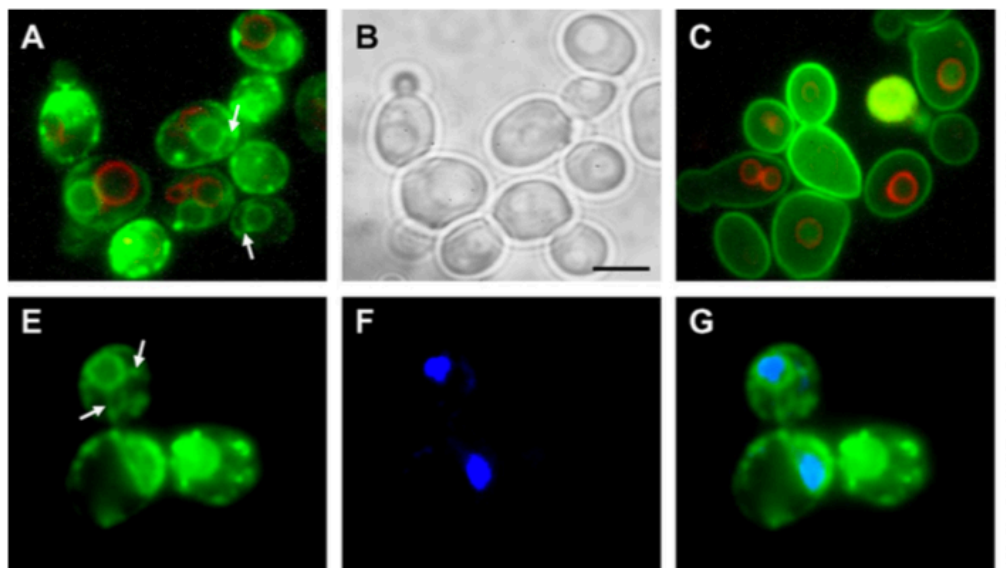

Fig. 3. ER membrane visualization of the HcZnT1:GFP fusion protein in yeast by epifluorescence microscopy. Cells were grown in SD medium and stained with FM4-64 (A HcAMT1:GFP (F). Different images from the same cells are shown: GFP fluorescence (A, C, and E), and bright field (B, D, and H) images from HcZnT1:GFP (A, B and E-H) or nucleus to peripheral ER structures. Bars $=5 \mathrm{~lm}$.

\subsection{Expression patterns of HcZnT1}

Transcript levels were monitored by RT-PCR on H. cylindrospo- rum mycelia grown at increasing concentrations of Zn or in different types of mycelia (Fig. 4). Increasing the level of $\mathrm{Zn}$ in the growth medium did not significantly change HcZnT1 transcript lev- els (Fig. 
4A, upper panel). A similar pattern was obtained with the control gene HcGS encoding the glutamine synthetase (Fig. 4A, lower panel). HcZnT1 transcripts were also detected in mycor- rhiza-associated mycelium and in sporocarps (Fig. 4B), indicating that HcZnT1 probably plays a crucial role in $\mathrm{Zn}$ homeostasis, being expressed in various fungal structures.

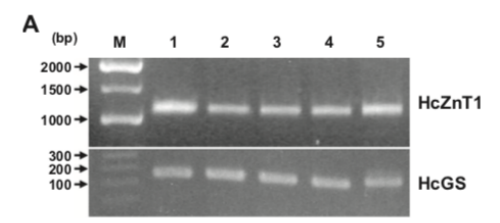

B

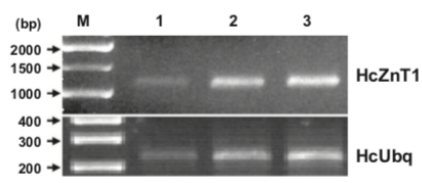

Fig. 4. Expression levels of $\mathrm{HcZnT1}$ in $\mathrm{H}$. cylindrosporum. Transcript levels at increasing $\mathrm{Zn}$ concentration (A) or in different fungal tissues (B) were estimated by RT-PCR. Expression level of HcZnT1 was compared to that of H. cylindrosporum glutamine synthetase (HcGS) or ubiquitin (HcUbq). In A, Zn concentrations are 0.3 (1), 3 (2), 30 (3), 300 (4) and 3000 (5) lM. In B, RT-PCR reactions were performed on mycorrhizal roots (1), sporocarps (2) and pure culture mycelia (3). Experiments were repeated three times and representative gels are shown.

\subsection{Fluorescence detection of zinc localization using zinquin}

Accumulation of zinc by H. cylindrosporum was further investigated by using fluorescence detection microscopy. The $\mathrm{Zn}$ concentration used here (200 lM) was chosen from preliminary experiments showing no effects on fungal growth. Zinquin labeled numerous small punctuate vesicles in H. cylindrosporum mycelia. This fluorescence pattern was identical whatever the age/position of the cell in the hypha (Fig. 5B and F). Consequently it seems very unlikely that this fluorescence pattern coincide with vacuoles. In order to verify this statement, we analysed vacuoles using a stain- ing procedure with carboxy-DCFDA, a specific marker of the vacu- olar lumen, at different positions in the hyphae of H. cylindrosporum. As previously reported by Fricker et al. (2008), who identified the vacuolar system of Phanerochaete velutina hy- phae with the same procedure, the structure of the vacuole devel- ops from a reticulum of fine tubes interspersed with small spherical vacuoles at the hyphal tip (Fig. 5A) to a series of small and larger spherical vacuoles in the older parts of the hypha (Fig. 5E). In both cases, the zinquin-derived fluorescence did not co-localize with that of the carboxy-DCFDA labeled vacuoles (Fig. 5D and H), demonstrating that zinquin-bound $\mathrm{Zn}$ pools are not localized into vacuoles.
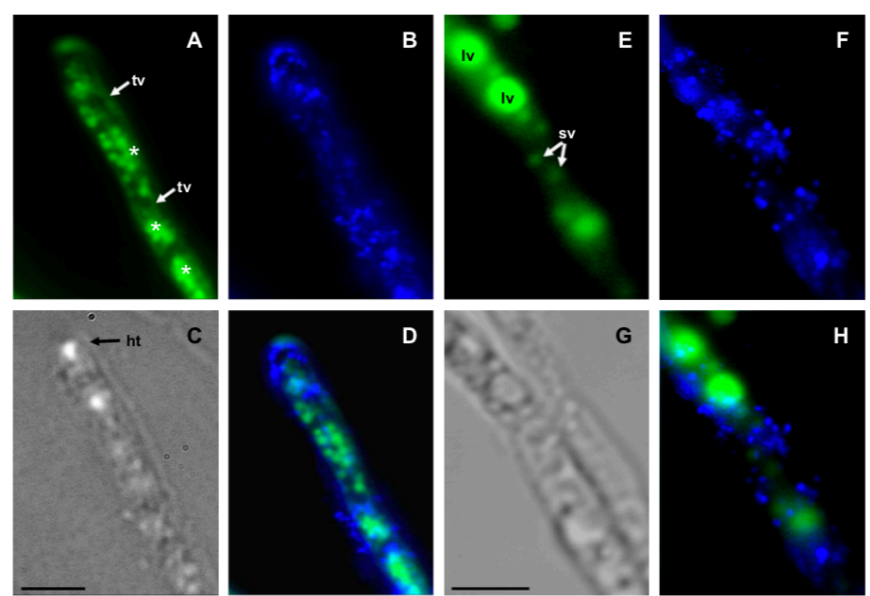

Fig. 5. Fluorescence microscopy images showing $\mathrm{Zn}$ localization in $\mathrm{H}$. cylindrosporum cells exposed to $200 \mathrm{~lm} \mathrm{ZnCl}_{2}$. Two representative parts [the apical region (A-D) and the proximal region (E-H)] of an individual hypha are shown. Zinquin fluorescent marker for zinc localization is shown in cyan (B and F). Control staining of vacuoles with the fluorescent marker carboxy-DCFDA is shown in green (A and E). Fig. 5D and 5H are zinquin + carboxy-DCFDA merged pictures from 5A and 5B, and 5E and 5F, respectively. tv: fine tubular vacuoles, sv: small spherical vacuoles, lv: large spherical vacuoles, ht: hyphal tip. The asterisks denote patches of small spherical vacuoles. Bars = $5 \mu \mathrm{m}$.

Table 1

Characterized fungal members of the CDF family that at least transport zinc.

\begin{tabular}{lllll}
\hline Gene & Substrate & Organism & Localization & Reference \\
\hline HcZnt1 & $\mathrm{Zn}$ & H. cylindrosporum & ER & Present study \\
Cis4 & $\mathrm{Zn}$ & S. pombe & Golgi & Fang et al. (2008) \\
GiZnT1 & (Zn) & G. intraradices & - & Gonzalez-Guerrero et al. (2005) \\
Zhf1 & $\mathrm{Zn}(\mathrm{Co}, \mathrm{Cd})$ & S. pombe & ER & Clemens et al. (2002) \\
Mcs2 & $\mathrm{Zn}$ & S. cerevisiae & ER/nucleus & Ellis et al. (2004), Li and Kaplan (2001) \\
Zrg17 & $\mathrm{Zn}$ & S. cerevisiae & ER/Golgi & Ellis et al. (2005), Yuan (2000) \\
Zrc1 & $\mathrm{Zn}(\mathrm{Cd})$ & S. cerevisiae & Vacuole & Kamizono et al. (1989), MacDiarmid et al. (2000) \\
Cot1 & Co, Zn & S. cerevisiae & Vacuole & Conklin et al. (1992), MacDiarmid et al. (2000) \\
\hline
\end{tabular}




\section{Discussion}

Ectomycorrhizal fungi, including Hebeloma species, have re- ceived considerable attention for use as efficient symbionts to im- prove mineral nutrition (Muller et al., 2007; Smith and Read, 2008) and to alleviate TE toxicity of their host plants (Adriaensen et al., 2006; Krpata et al., 2008; Krupa and Kozdroj, 2007; Krznaric et al., 2009; Meharg and Cairney, 2000; Roy et al., 2007). Less infor- mation is available about the capability of ectomycorrhizal fungi to actively accumulate TE.

In the present study, we identified a transporter gene involved in zinc homeostasis, HcZnT1, which falls into the zinc-CDF group according to the CDF classification (Montanini et al., 2007). This group includes the Zrc1-like cluster enclosing only fungal CDF originating from Ascomycetes, Basidiomycetes, and Zygomycetes. The present work confirms that this CDF group, referred to as the $\mathrm{Zn}$-CDF group, contained characterized members that at least transport zinc (Table 1). Members of the CDF family typically con- tain conserved histidine-rich regions in the intracellular loop of the protein between transmembrane domains 4 and 5 (Montanini et al., 2007). These are postulated to facilitate $\mathrm{Zn}^{2+}$ binding (Bloss et al., 2002; Montanini et al., 2007). Consistent with this observa- tion, we found a total of 17 histidine residues, 7 of which were con- served. Nine of these were located between transmembrane domains 4 and 5. CDF transporters are postulated to be involved exclusively in transport of metals into intracellular vesicles/com- partments or out of the cell across the plasma membrane (Palmiter and Huang, 2004). The HxxxD motif typical for Zn-specific CDF members (Montanini et al., 2007) were also detected in HcZnT1, confirming its putative specificity towards zinc. The zinc specificity was confirmed by complementation tests using a range of yeast mutants. Data obtained with the different mutant strains suggest that HcZnT1 specifically transports $\mathrm{Zn}$ but not Co, Mn, nor Cd.

HcZnT1was constitutively expressed in the mycelium whatever the Zn concentration applied to the medium. Conversely, GintZnT1 was slightly up-regulated in the extraradical mycelium of G. intraradices by 75lM Zn after 6 or $12 \mathrm{~h}$ of incubation (Gonzalez-Guerrero et al., 2005). Although also in our study was used a short incubation time (18 h) any changes in HcZnT1 were observed (Fig. 4). A shorter time of incubation ( $6 \mathrm{~h}$ ) did neither re- sult in a change of expression (data not shown). However, fungal and plant CDFs of the Zn group are usually not regulated by high Zn concentrations (Blaudez et al., 2003). Similarly, Zrc1 was not regulated by high Zn (MacDiarmid et al., 2003) suggesting that the protein is primarily involved in Zn homeostasis under normal conditions and may act as a crucial response element against Zn toxicity when cells are exposed to high concentrations of this ele- ment. Zrc1 was yet regulated during Zn-shock after Zn-deficiency (MacDiarmid et al., 2003).

We tried to increase the expression level of HcZnT1 in H. cylin- drosporum in order to possibly obtain a phenotype with an in- creased Zn tolerance. For this purpose, we cloned the HcZnT1 cDNA in the pFAT3 binary vector and transformed Agrobacterium tumefaciens cells that were subsequently used in cocultures with Hebeloma mycelia, according to a method described earlier (Bellion et al., 2007). Hebeloma transformants expressed this supplemental copy of HcZnT1 as revealed by RT-PCR but were not significantly more tolerant to $\mathrm{Zn}$ when compared to control transformants (col- onies transformed with the empty vector) or wild-type colonies (data not shown). Reduction of HcZnT1 expression level would be the reverse strategy to get additional information on the function of this gene; however the production of targeted KO mutants in ectomycorrhizal fungi is not yet feasible due to the strong preva- lence of illegitimate recombination over homologous recombina- tion in these organisms. Similarly, RNAi or antisense technologies have not yet been successfully applied to Hebeloma and other mycorrhizal fungi, except for L. bicolor (Kemppainen et al., 2009).

When compared to previously characterized CDF members (Fig. 1 and Table 1), HcZnT1 was mostly similar to the vacuolar membrane located transporters Zrc1 and Cot1 from S. cerevisiae that clustered together with the ER localized Zhf1 from S. pombe (Montanini et al., 2007) and to the G. intraradices sequence (Gonz- alez-Guerrero et al., 2005). Apart from yeast, no full characteriza- tion of CDF members was reported in other fungal subphyla. The Glomeromycete GintZnT1 protein was proposed to be involved in Zn homeostasis (Gonzalez-Guerrero et al., 2005). However, it did not confer Zn tolerance when expressed in yeast mutants and its localization was not reported. To gain further information on the role of HcZnT1 in Zn homeostasis/tolerance, we investigated its cellular localization when expressed in yeast. HcZnT1::GFP fluores- cent pattern was typical of ER targeting (Fig. 3A, E, and G), with ER structures surrounding the nucleus (as confirmed by DAPI staining of nuclei, Fig. 3G) and cytoplasmic strands connecting the nucleus to peripheral ER structures.

It is thus tempting to speculate that HcZnT1 is located on ER membranes, as shown for the S.pombe Zhf1 (Clemens et al., 2002), and participate to Zn loading in this compartment. From this line of evidence, two hypotheses arise concerning the role of HcZnT1. First HcZnT1 may have a general role in Zn homeostasis under normal conditions by providing Zn to zinc-requiring pro- teins that are processed in the ER. Second HcZnT1 could also be in- volved in Zn tolerance under Zn stress conditions by loading Zn ions into this subcellular compartment and consequently by con- tributing to the detoxification of the cytosol. This mechanism could resemble that of S. pombe in which the ER-located zinc transporter Zhf1 plays an important role in Zn tolerance and the ER is serving as a major site for Zn storage (Clemens et al., 2002). Interestingly, when investigating the genome sequence of this organism, we found that Zhf1 is the only member of the Zn-CDF gene subfamily (Blaudez, unpublished data). This ER-mediated strategy in transi- tion metal cell biology in S. pombe differed from that employed by S. cerevisiae where the vacuole is the main site of Zn storage (Simm et al., 2007) and where the two vacuolar Zn-CDF transport- ers Zrc1p and Cot1p play essential role in Zn sequestration and tol- erance (Eide, 2006). The next step will be to develop tools such as KO or RNAi mutant lines in Hebeloma, tools necessary to gain more comprehensive details on the role of HcZnT1 in Zn homeostasis/ tolerance. The coming sequencing of the H. cylindrosporum genome should also reveal other putative key determinants of $\mathrm{Zn}$ homeo- stasis/tolerance.

As another step forward into the understanding of $\mathrm{Zn}$ homeo- stasis in Hebeloma, we also investigated $\mathrm{Zn}$ pools in hyphae by using zinquin, a Zn-specific fluorescent probe. When grown at a sub-lethal Zn concentration, H. cylindrosporum hyphae after zin- quin staining showed intracellular zinc pools located in small punctuate cytoplasmic vesicles, but not into vacuolar compart- ments. These findings are in agreement with previous studies based on microscopy analyses and quantitative measurements by X-ray microanalysis, showing that extracellular binding and cyto- solic sequestration of Zn occurred in Hebeloma crustuliniforme cells, whereas vacuoles were presumably not a significant $\mathrm{Zn}$ storage compartment in this fungus (Frey et al., 2000). 
The vesicles that we identified in $\mathrm{H}$. cylindrosporum hyphae are similar to the zincosomes described in mammalian cells (Wellenreuther et al., 2009) and to cytoplasmic vesicles reported in yeast (Li and Kaplan, 2001; Devirgiliis et al., 2004), although the identity of these zincosomes is still unknown. Colocalization studies showed that these vesicles do not correspond to vacuoles, prevac- uolar compartments, nor to Golgi structures (Eide, 2006). The pres- ent study similarly ruled out the vacuolar nature of these Zn- containing vesicles in the filamentous fungus $\mathrm{H}$. cylindrosporum. In- deed the zinquin-derived fluorescence pattern of these vesicles did not colocalize with the carboxy-DCFDA-derived fluorescence pat- tern identifying vacuoles. The putative functions of these zinco- somes have been discussed by Eide (2006), who hypothesized that they could serve to buffer cytosolic Zn from transient pertur- bations much like the vacuole in yeast or to act as a storage com- partment for $\mathrm{Zn}$ that can be later remobilized under $\mathrm{Zn}$ deficiency.

However, the lack of zinquin fluorescence in vacuoles has to be interpreted with caution since zinquin binding is impaired in the vacuole by the acidic $\mathrm{pH}$ that is actively maintained inside this compartment. Similarly, vacuolar Zn-ligand complexes could also impair zinquin binding to $\mathrm{Zn}^{2+}$. However, the present study clearly identifies a vesicular compartment of free $\mathrm{Zn}$ that does not corre- spond to vacuoles and that may represent the main pool of free $\mathrm{Zn}$ ions in $\mathrm{H}$. cylindrosporum. Further microscopic observations will have to be performed to identify other potential pools of $\mathrm{Zn}$ in Heb- eloma and in other mycorrhizal fungi.

The approaches used in the present paper highlight the putative function of intracellular compartments other than vacuoles in zinc tolerance/homeostasis in H. cylindrosporum cells. In order to know whether the vesicular compartment (or zincosome) evidenced by zinquin is linked to the ER compartment (hosting HcZnT1) or not, future lines of research should focus on the determination of the exact nature of these $\mathrm{Zn}$-containing vesicles.

Mycorrhizal fungi are important for host protection against the toxic effects of trace elements (Baum et al., 2006 ; Bellion et al., 2006 ; Kozdroj et al., 2007; Krznaric et al., 2009). Particularly, me- tal tolerance mechanisms would play a crucial role in the TE biofil- tering capacity of fungal cells. Therefore on TE-contaminated soils, the mycorrhizal symbiosis would favor both the establishment of trees but also the production of woody biomass with a reduced TE content, that could subsequently be used in different bioenergy pathways (Khasa et al., 2002 ; Sell et al., 2005).

\section{Acknowledgments}

We thank Roland Marmeisse (Lyon, France) and Hervé Sentenac (Montpellier, France) for kindly providing us with Hebeloma sporocarps and the cDNA library, respectively. This work was partly sup- ported by grants from the PNETOX programme, the ANR Eumetatox Project (06-BLAN-0088), and the Région Lorraine.

\section{References}

Adriaensen, K. et al., 2006. Zinc-tolerant Suillus bovinus improves growth of Zn- exposed Pinus sylvestris seedlings. Mycorrhiza 16, 553-558.

Anton, A. et al., 1999. CzcD is a heavy metal ion transporter involved in regulation of heavy metal resistance in Ralstonia sp. strain CH34. J. Bacteriol. 181, 6876-6881. Arriagada C. et al., 2010. Improved zinc tolerance in Eucalyptus globulus inoculated with Glomus deserticola and Trametes versicolor or Coriolopsis rigida. Soil Biol. Biochem. 42, 118-124. Baum, C. et al., 2006. Heavy-metal mobilization and uptake by mycorrhizal and nonmycorrhizal willows (Salix x dasyclados). J. Plant Nutr. Soil Sci. 169, 516- 522. Bellion, M. et al., 2006. Extracellular and cellular mechanisms sustaining metal tolerance in ectomycorrhizal fungi. FEMS Microbiol. Lett. 254, 173-181. Bellion, M. et al., 2007. Metal induction of a Paxillus involutus metallothionein and its heterologous expression in Hebeloma cylindrosporum. New Phytol. 174, 151- 158. Bissonnette, L. et al., 2009. Phytoextraction of heavy metals by two Salicaceae clones in symbiosis with arbuscular mycorrhizal fungi during the second year of a field trial. Plant Soil 1,13 .

Blaudez, D. et al., 2000a. Effects of heavy metals on nitrogen uptake by Paxillus involutus and mycorrhizal birch seedlings. FEMS Microbiol. Ecol. 33, 61-67. Blaudez, D. et al., Blaudez, D. et al., 2000a. Effects of heavy metals on nitrogen uptake by Paxillus involutus and mycorrhizal birch seedlings. FEMS Microbiol. Ecol. 33, 61-67. Blaudez, D. et al.,
2000b. Cadmium uptake and subcellular compartmentation in the ectomycorrhizal fungus Paxillus involutus. Microbiology 146, 1109-1117. Blaudez, D. et al., 2003. Poplar metal tolerance protein 1 confers zinc tolerance and is an oligomeric vacuolar zinc transporter with an essential leucine zipper motif. Plant Cell 15, 2911-2928. tolerance protein 1 confers zinc tolerance and is an oligomeric vacuolar zinc transporter with an essential leucine zipper motif. Plant Cell 15, $2911-2928$.
Bloss, T. et al., 2002. Characterization of the ZAT1p zinc transporter from Arabidopsis thaliana in microbial model organisms and reconstituted proteoliposomes. Planta 214 , 783-791.

Carvalho, L.M. et al., 2006. Arbuscular mycorrhizal fungi enhance root cadmium and copper accumulation in the roots of the salt marsh plant Aster tripolium L. Plant Soil 285, 161-169.

Christie, P. et al., 2004. Arbuscular mycorrhiza can depress translocation of zinc to shoots of host plants in soils moderately polluted with zinc. Plant Soil 261, $209-217$. Clemens, S. et al., 2002. A transporter in the endoplasmic reticulum of Schizosaccharomyces pombe cells mediates zinc storage and differentially affects transition metal tolerance. J. Biol. Chem. 277, 18215-18221.

Cobbett, C., Goldsbrough, P., 2002. Phytochelatins and metallothioneins: roles in heavy metal detoxification and homeostasis. Annu. Rev. Plant Biol. 53, 159-182. Colpaert, J.V. et al., 2004. Evolutionary adaptation to Zn toxicity in populations of Suilloid fungi. New Phytol. 162, 549-559.Conklin, D.S. et al., 1992. COT1, a gene involved in cobalt accumulation in Saccharomyces cerevisiae. Mol. Cell. Biol. 12,3678-3688.

Courbot, M. et al., 2004. Cadmium-responsive thiols in the ectomycorrhizal fungus Paxillus involutus. Appl. Environ. Microbiol. 70, 7413-7417.

Devirgiliis, C. et al., 2004. Exchangeable zinc ions transiently accumulate in a vesicular compartment in the yeast Saccharomyces cerevisiae. Biochem. Biophys. Res. Commun.

323, 58-64.

Eide, D.J., 2006. Zinc transporters and the cellular trafficking of zinc. Biochim. Biophys. Acta 1763, 711-722.

Eide, D.J., 2006. Zinc transporters and the cellular trafficking of zinc. Biochim. Biophys. Acta 1763, 711-722.
Ellis, C.D. et al., 2004. Zinc and the Msc2 zinc transporter protein are required for endoplasmic reticulum function. J. Cell Biol. 166, 325-335.

Ellis, C.D. et al., 2005. Heteromeric protein complexes mediate zinc transport into the secretory pathway of eukaryotic cells. J. Biol. Chem. 280, 28811-28818. Fang, Y. et al., 2008. Cation diffusion facilitator cis4 is implicated in Golgi membrane trafficking via regulating zinc homeostasis in fission yeast. Mol. Biol. Cell 19, 1295-1303.

Frey, B. et al., 2000. Extracellular complexation of Cd in the Hartig net and cytosolic Zn sequestration in the fungal mantle of Picea abies - Hebeloma crustuliniforme ectomycorrhizas. Plant Cell Environ. 23, 1257-1265.

Fricker, M.D. et al., 2008. Imaging complex nutrient dynamics in mycelial networks. J. Microsc. 231, 317-331.

Gadd, G.M., 2010. Metals, minerals and microbes: geomicrobiology and bioremediation. Microbiology 156, 609-643.

Gaither, L.A., Eide, D.J., 2001. Eukaryotic zinc transporters and their regulation. Biometals 14, 251-270.

Gietz, D. et al., 1992. Improved method for high-efficiency transformation of intact yeast-cells. Nucleic Acids Res. 20,1425.

Gonzalez-Guerrero, M. et al., 2005. Characterization of a Glomus intraradices gene encoding a putative Zn transporter of the cation diffusion facilitator family. Fungal Genet. Biol. 42, 130-140.

Hall, J.L., Williams, L.E., 2003. Transition metal transporters in plants. J. Exp. Bot. 54, 2601-2613

Hildebrandt, U. et al., 2007. Arbuscular mycorrhiza and heavy metal tolerance. Phytochemistry 68, 139-146.

Jacob, C. et al., 2001. Molecular cloning, characterization and regulation by cadmium of a superoxide dismutase from the ectomycorrhizal fungus Paxillus involutus. Eur. I.

Biochem. 268, 3223-3232.

Javelle, A. et al., 2003. Molecular characterization, function and regulation of ammonium transporters (Amt) and ammonium-metabolizing enzymes (GS, NADP-GDH) in the ectomycorrhizal fungus Hebeloma cylindrosporum. Mol. Microbiol. 47, 411-430.

Kambe, T. et al., 2004. Overview of mammalian zinc transporters. Cell. Mol. Life Sci. 61, 49-68.

Kamizono, A. et al., 1989. Identification of a gene conferring resistance to zinc and cadmium ions in the yeast Saccharomyces cerevisiae. Mol. Gen. Genet. $219,161-167$. Kemppainen, M. et al., 2009. RNA silencing in the model mycorrhizal fungus Laccaria bicolor: gene knock-down of nitrate reductase results in inhibition of symbiosis with Populus. Environ. Microbiol. 11, 1878-1896.

Khasa, P.D. et al., 2002. The mycorrhizal status of selected poplar clones introduced in Alberta. Biomass Bioenergy 22, 99-104.

Kozdroj, J. et al., 2007. Mycorrhizal fungi and ectomycorrhiza associated bacteria isolated from an industrial desert soil protect pine seedlings against Cd(II) impact.

Ecotoxicology 16, 449-456. 
Krpata, D. et al., 2008. Ectomycorrhizal communities associated with Populus tremula growing on a heavy metal contaminated site. Mycol. Res. 112, 1069- 1079. Krupa, P., Kozdroj, J., 2004. Accumulation of heavy metals by ectomycorrhizal fungi colonizing birch trees growing in an industrial desert soil. World J. Microbiol. Biotechnol. 20, 427-430.

Krupa, P., Kozdroj, J., 2007.

Soil Pollut 182, $83-90$

Krznaric, E. et al, 2009. Cd-tolerant Suillus luteus: a fungal insurance for pines exposed to Cd. Environ. Pollut 157, 1581-1588.

Kuffner, M. et al., 2008. Rhizosphere bacteria affect growth and metal uptake of heavy metal accumulating willows. Plant Soil 304, 35-44.

Lambilliotte, R. et al., 2004. Large-scale identification of genes in the fungus Hebeloma cylindrosporum paves the way to molecular analyses of ectomycorrhizal symbiosis. New Phytol. 164, 505-513.

Lee, R. et al., 2003. Zinc accumulation in N-methyl-N-nitrosourea-induced rat mammary tumors is accompanied by an altered expression of ZnT-1 and metallothionein. Exp. Biol. Med. 228, 689-696.

Leung, H.M. et al., 2007. Survival strategies of plants associated with arbuscular mycorrhizal fungi on toxic mine tailings. Chemosphere 66, 905-915.

Li, L., Kaplan, J., 1997. Characterization of two homologous yeast genes that encode mitochondrial iron transporters. J. Biol. Chem. 272, 28485-28493

Li, L., Kaplan, J., 1998. Defects in the yeast high affinity iron transport system result in increased metal sensitivity because of the increased expression of transporters with a

broad transition metal specificity. J. Biol. Chem. 273, 22181-22187.

Li, L., Kaplan, J., 2001. The yeast gene MSC2, a member of the cation diffusion facilitator family, affects the cellular distribution of zinc. J. Biol. Chem. 276, 5036-5043. MacDiarmid, C.W. et al., 2000. Zinc transporters that regulate vacuolar zinc storage in Saccharomyces cerevisiae. EMBO J. 19, 2845-2855.

MacDiarmid, C.W. et al,, 2003. Induction of the ZRC1 metal tolerance gene in zinc- limited yeast confers resistance to zinc shock. J. Biol. Chem. 278, 15065-15072. Mahadevan, I.B. et al., 1996. The synthesis of zinquin ester and zinquin acid, zinc(II)-specific fluorescing agents for use in the study of biological zinc(II). Aust. J. Chem. 49, 561-568.

Maser, P. et al., 2001. Phylogenetic relationships within cation transporter families of Arabidopsis. Plant Physiol. 126, 1646-1667.

Maser, P. et al., 2001. Phylogenetic relationships within cation transporter families of Arabidopsis. Plant Physiol. 126, 1

Meharg, A.A., Cairney, J.W.G., 2000. Ectomycorrhizas - extending the capabilities of rhizosphere remediation? Soil Biol Biochem. 32, $1475-1484$.

Montanini, B. et al., 2007. Phylogenetic and functional analysis of the Cation Diffusion Facilitator (CDF) family: improved signature and prediction of substrate specificity. BMC Genomics 8,107

Muller, T. et al., 2007. Nitrogen transport in the ectomycorrhiza association: the Hebeloma cylindrosporum-Pinus pinaster model. Phytochemistry 68, 41-51. Nies, D.H., 2003. Efflux-mediated heavy metal resistance in prokaryotes. FEMS Microbiol. Rev. 27, 313-339.

Nies, D.H., Silver, S., 1995. Ion efflux systems involved in bacterial metal resistances. J. Ind. Microbiol. 14, 186-199.

Palmiter, R.D., Huang, L., 2004. Efflux and compartmentalization of zinc by members of the SLC30 family of solute carriers. Pflugers Arch. 447, 744-751. Paulsen, I.T., Saier Jr.,

M.H., 1997. A novel family of ubiquitous heavy metal ion transport proteins. J. Membr. Biol. 156, 99-103.

Ramesh, G. et al., 2009. Different patterns of regulation for the copper and cadmium Metallothioneins of the ectomycorrhizal fungus Hebeloma cylindrosporum. Appl. Environ Microbiol. 75, 2266-2274.

Roy, S. et al., 2007. Combining alders, frankiae, and mycorrhizae for the revegetation and remediation of contaminated ecosystems. Can. J. Bot. 85, $237-251$.

Sell, J. et al., 2005. Contribution of ectomycorrhizal fungi to cadmium uptake of poplars and willows from a heavily polluted soil. Plant Soil 277, 245-253. Simm, C. et al., 2007. Saccharomyces cerevisiae vacuole in zinc storage and intracellular zinc distribution. Eukaryot. Cell 6, 1166-1177.

Smith, S.E., Read, D.J., 2008. Mycorrhizal Symbiosis. Academic Press, London, pp. 800 .

Stommel, M. et al., 2001. EST-library construction using spore RNA of the arbuscular mycorrhizal fungus Gigaspora rosea. Mycorrhiza 10, $281-285$.

Ton, V.K. et al., 2002. Functional expression in yeast of the human secretory pathway $\mathrm{Ca}^{2+}, \mathrm{Mn}^{2+}$-ATPase defective in Hailey-Hailey disease. J. Biol. Chem. $277,6422-6427$. Vallino, M. et al., 2009. Cu, $\mathrm{Zn}$ superoxide dismutase and zinc stress in the metal- tolerant ericoid mycorrhizal fungus Oidiodendron maius Zn. FEMS Microbiol. Lett. 293 , 48-57. Vida, T.A., Emr, S.D., 1995. A new vital stain for visualizing vacuolar membrane dynamics and endocytosis in yeast. J. Cell Biol. 128, 779-792.

Wei, Y., Fu, D., 2005. Selective metal binding to a membrane-embedded aspartate in the Escherichia coli metal transporter YiiP (FieF). J. Biol. Chem. 280, 33716- 33724.

Wellenreuther, G. et al., 2009. The ligand environment of zinc stored in vesicles. Biochem. Biophys. Res. Commun. 380, 198-203.

Yuan, D.S., 2000. Zinc-regulated genes in Saccharomyces cerevisiae revealed by transposon tagging. Genetics 156, 45-58. 